\title{
The Study of Feasibility and Acceptability of using HIV Self- Tests in High-Risk Iranian Populations (FSWs, MSM, and TG Populations): A Cross-Sectional Study
}

\section{Ghobad Moradi}

Kurdistan University of Medical Science: Kurdistan University of Medical Sciences

\section{Elnaz Ezzati Amini}

Kurdistan University of Medical Science: Kurdistan University of Medical Sciences

\section{Azam Valipour}

Tehran University of Medical Sciences

\section{Katayoon Tayeri}

Tehran University of Medical Sciences

\section{Parvin Afsar Kazerooni}

Tehran University of Medical Sciences

\section{Leila Molaeipour}

Iran University of Medical Sciences: Tehran University of Medical Sciences

Yousef Moradi ( $\square$ yousefmoradi211@yahoo.com )

Kurdistan University of Medical Science: Kurdistan University of Medical Sciences https://orcid.org/0000-0002-2936-5930

\section{Research}

Keywords: HIV self-test, female sex worker, transgender, men who have sex with men, high-risk population, Iranian.

Posted Date: November 16th, 2021

DOI: https://doi.org/10.21203/rs.3.rs-1071943/v1

License: (c) (i) This work is licensed under a Creative Commons Attribution 4.0 International License. Read Full License 


\section{Abstract}

Background: This study aimed to evaluate the feasibility of using the HIV Self-Test in high-risk Iranian groups (MSM, FSWs, and TG people).

Methods: This study was conducted in 2 main steps (the pre and post HIVST intervention and the qualitative method) between 1 October 2020 and 22 June 2021 in Tehran, Iran. The sample size needed for this study generally was 1000 people, including FSWs, MSM, and transgender individuals. The sampling method in this study was convenience sampling, while snowball sampling was used to complete the number of samples.

Results: A total of 930 eligible respondents were enrolled in the study, of whom 456 (49\%) were female, 49 (5.3\%) were transgender ( $98 \%$ of TGs were male to female). The median age was $33.63 \pm 10.54$ SD. The feasibility of the use of HIV selftests in Iranian high-risk groups was significantly high. The majority of participants (97\%) did not have any confidentiality problems while preparing or performing the test. In general, feasibility was assessed based on five questions. The overall feasibility score was 6.33 (0.824 SD). Taking tests, reading HIV test results, finding a safe place to do the test, and accessing HIV self-testing showed a high average.

Conclusion: HIV self-testing was highly acceptable and feasible to permit routine HIV testing between high-risk populations efficiently.

\section{Introduction}

Improving the diagnosis helps achieve national/global goals of HIV epidemic control [1]. The Islamic Republic of Iran, in all four UN special meetings on HIV in 2003, 2008, 2011, and 2016 has committed itself to HIV control and has signed the declarations of these meetings. At the 2016 summit, the UN member countries and Iran pledged, based on previous announcements, to accelerate the fight against AIDS through shortcuts to end the epidemic by 2030 . These fast-track routes were divided into ten sections, the first of which was identifying patients and ensuring the access of HIV-infected people to treatment based on 90 , 90,90 strategies by 2020 [2-6]. Therefore, implementing a fast and safe way without stigma and discrimination to identify cases in high-risk groups can help achieve the ten quick ways to end the HIV epidemic [7, 8]. Different diagnostic technologies in rapid diagnostic methods can help improve the diagnosis and ultimately control the disease. In March 2004, the HIV Antibody Rapid Detection Kit, introduced by the US Food and Drug Administration for salivary fluid samples in all individuals, became the first HIV Self-Test, or home testing [9-12].

In the following years, the quality of this test gradually improved, and its diagnostic value was also significantly enhanced. In Iran, with the control of the epidemic in cases of Injecting Drug Users (IDUs) and prisoners with the method of harm reduction, the transmission has led to high-risk groups. Of course, this also happened in many countries around the world. Given that most people at risk or high-risk groups, such as men who have sex with men (MSM), female sex workers (FSWs), and transgender people (TGs), may have not even once been diagnosed with HIV, their access to these tests is of great importance $[8,13,14]$. Many of these key groups may be infected with HIV, but they are unaware of their status, so diagnosing the disease in these people can prevent the transmission of the infection to others. One of the reasons for not performing diagnostic tests by high-risk people may be the lack of easy access to these tests [15]. Therefore, by increasing the availability of the HIV SelfTest (HIVST) for high-risk groups, effective action can be taken in the early detection of the disease in high-risk individuals to prevent the transmission of the disease to others $[16,17]$. Even this program can reduce the social stigma and fear in people of visiting centers and create more confidence in these groups to help the diagnosis process of HIV infection. In pursuit of these goals, more key groups will receive HIV / AIDS care and treatment [18-20]. If this program is accepted, the possibility of HIV testing for individuals and their sexual partners who may have never been tested for HIV will be provided and increased. Many countries, including Iran, have accepted this policy, and some countries have even implemented it. Iran seeks to upgrade a codified action plan for feasibility and operationalizing HIVST in the general population and high-risk groups. This study aimed to evaluate the feasibility of using the HIV Self-Test in high-risk Iranian groups (MSM, FSWs, and TG people). It is expected that the results of this study can provide valuable evidence for the proper implementation of this program in the country.

Page 2/18 


\section{Materials And Methods}

This study was conducted in 2 main steps (the pre and post HIVST intervention and the qualitative method) between 1 October 2020 and 22 June 2021 in Tehran, Iran. This study has been received the national ethical code (Ethical code: IR.MUK.REC.1399.133). Also, all participants (MSM, FSWs, and TGs) signed an informed consent form.

A pre and post HIVST intervention was conducted. The target population in this study was FSWs, MSM, and TGs with their sexual partners. After explaining the study's objectives to the target population, they were encouraged to invite their sexual partners to participate in pre and post HIVST intervention studies.

The sample size needed for this study generally was 1000 people include FSWs and their sexual partners (400 people), MSM and their sexual partners (400 people), and transgender individuals and their sexual partners (200 people). The sampling method in this study was convenience sampling, while snowball sampling was used to complete the number of samples. In this way, after the workshop, qualified communicators encouraged their fellow citizens to participate in this study. In each of the selected centers ( 9 centers), each of the consultants was linked to 10-15 communicators. The inclusion criteria included lack of knowledge of key people from their status of HIV/AIDS, age over 18 years, satisfaction to participate in the study, men who have had at least one sex with other men during the last 12 months (vulnerable men (MSM) and their sexual partners), women who have had at least one sex in the past 12 months in exchange for money (vulnerable women (FSWs)), men who have had at least one sex with vulnerable women (FSWs) during the last 12 months (sexual partners of vulnerable women (FSWs)), people who were diagnosed transgender by communicators (transgender people) and those who have had at least one sex with transgender individuals over the past 12 months (sexual partners of transgender people).

Before starting the study, counseling and testing centers, consultants, communicators, and HIV self-test kits were provided and prepared. Also, training packages and services were provided for the key groups under study, and planning for holding workshops was done. Then, holding seminars, distribution of equipment and items required at selected centers, sampling and collecting data, following up cases with positive test results for connecting them to the chosen centers (to receive a consultation, conduct confirmation tests and start treatment), and performing qualitative interviews also were planned and performed.

To achieve the research objectives, proportional tools and methods of collecting data were used in different stages of the study. The first part was "the questionnaire of demographic characteristics of key populations participating in the study," the second part was "the questionnaire of knowledge and awareness of key groups participating in the study before performing the test," the third part was "the questionnaire of acceptability, feasibility, and satisfaction" and the fourth part was "the questionnaire of semi-structured interviews on identifying the benefits and disadvantages of the test and determining challenges in using this test in service provision centers."

In the first three sections, to collect data and daily reports, online questionnaire-making software was used. In the fourth section, the data were qualitatively collected through holding focused group discussions. This step was done after the third step. In the qualitative step, several subjects were selected from all three key populations of MSM, FSWs, and TGs. Focused group discussions were held with selected individuals to determine the test's advantages and disadvantages, the problems in performing the test, and the challenges in using the test in service centers.

Quality assurance is a set of activities before the study to collect the data with the highest possible quality. Also, all actions performed while collecting information or identifying and modifying any deviation from the executable guidelines are known as quality control. In this study, the following measures were used to ensure and control the quality of collected data: A standard executive protocol was developed, the authoritative administrative guide was evaluated using a pilot study, and an organizational observer was hired to monitor and assess the study process closely. Regular weekly meetings were held to review and resolve issues, and data cleansing was repeatedly performed.

\section{Statistical Analysis}


For the quantitative part of the study, the data obtained from questionnaires and checklists related to the level of Acceptance

and satisfaction were inserted into Excel. Then, relevant analyses such as analytical and descriptive analyses were performed using STATA version 16. After each qualitative interview, it was first transcribed into a Word file and was reviewed and evaluated. Then, if there were any ambiguous questions or problems in conducting the interview, the issues would be corrected and considered for the following discussion. If any questions helped achieve the study's objectives but had remained unanswered in the interviews, they would be added to the checklist for coming interviews. MAXQDA version 10 was used to analyze open categories and codes in each interview.

\section{Results}

A total of 930 eligible respondents were enrolled in the study, of whom $456(49 \%)$ were female, $49(5.3 \%)$ were transgender ( $98 \%$ of TGs were male to female), 804 (86\%) were single, 568 (61\%) were unemployed, 675 (73\%) were diploma and above, 629 (67.6\%) were living with a first-degree relative, and 174 (18.7\%) were living alone. The median age was $33.63 \pm 10.54$ SD (interquartile range [IQR]: 18-77), and 406 people (62.10\%) were less than 35 years old (Table 1). 
Socio-Demographic characteristics of respondents at baseline by key groups.

\begin{tabular}{|c|c|c|c|c|c|c|c|}
\hline & & Key groups & & & & & \\
\hline \multirow[t]{3}{*}{ Variables } & Total & FSW & $\begin{array}{l}\text { FSW Sexual } \\
\text { Partner }\end{array}$ & MSM & $\begin{array}{l}\text { MSM } \\
\text { Sexual } \\
\text { Partner }\end{array}$ & TG & $\begin{array}{l}\text { TG Sexual } \\
\text { Partner }\end{array}$ \\
\hline & \multirow[t]{2}{*}{ Number (\%) } & Number (\%) & Number (\%) & $\begin{array}{l}\text { Number } \\
(\%)\end{array}$ & $\begin{array}{l}\text { Number } \\
(\%)\end{array}$ & $\begin{array}{l}\text { Number } \\
\text { (\%) }\end{array}$ & $\begin{array}{l}\text { Number } \\
(\%)\end{array}$ \\
\hline & & $454(48.8)$ & $60(6.5)$ & $352(37.8)$ & $16(1.7)$ & $44(4.7)$ & $4(0.4)$ \\
\hline \multicolumn{8}{|l|}{ Location } \\
\hline Alborz & 208 (22.4) & $114(25.1)$ & $14(23.3)$ & 69 (19.6) & $2(12.5)$ & 8 (18.2) & $1(25.0)$ \\
\hline Tehran & 722 (77.6) & 340 (74.9) & 46 (76.7) & $283(80.4)$ & $14(87.5)$ & $36(81.8)$ & $3(75.0)$ \\
\hline Age $( \pm S D)$ & $33.63(10.54)$ & $39.19(10.15)$ & $35.22(12.02)$ & 27.79(6.54) & $27.00(7.43)$ & $23.59(4.16)$ & $30.75(3.78)$ \\
\hline \multicolumn{8}{|l|}{ Age } \\
\hline $18-25$ & $238(25.6)$ & $39(8.6)$ & $15(25.0)$ & $144(40.9)$ & $9(56.3)$ & $31(70.5)$ & $0(0.0)$ \\
\hline $26-35$ & $339(36.5)$ & 131(28.9) & $16(26.7)$ & $169(48.0)$ & $6(37.5)$ & 13(29.5) & $4(100.0)$ \\
\hline $36-45$ & 215(23.1) & 164(36.1) & 18(30.0) & $33(9.4)$ & $0(0.0)$ & $0(0.0)$ & $0(0.0)$ \\
\hline $46+$ & 138(14.8) & $120(26.4)$ & 11(18.3) & $6(1.7)$ & $1(6.3)$ & $0(0.0)$ & $0(0.0)$ \\
\hline \multicolumn{8}{|l|}{ Gender } \\
\hline Female & $456(49.0)$ & $454(100.0)$ & $2(3.3)$ & $0(0.0)$ & $1(6.3)$ & $0(0.0)$ & $0(0.0)$ \\
\hline Male & $425(45.7)$ & $0(0.0)$ & $58(96.7)$ & $349(99.1)$ & 14(87.5) & $0(0.0)$ & $3(75.0)$ \\
\hline Transgender & $49(5.3)$ & $0(0.0)$ & $0(0.0)$ & $3(0.9)$ & $1(6.3)$ & $44(89.8)$ & $1(25.0)$ \\
\hline \multicolumn{8}{|l|}{$\begin{array}{l}\text { Sex } \\
\text { Reassignment } \\
\text { Therapy }\end{array}$} \\
\hline $\begin{array}{l}\text { Male to } \\
\text { Female }\end{array}$ & $1(2.0)$ & $0(0.0)$ & $0(0.0)$ & $0(0.0)$ & 1(100.0) & $0(0.0)$ & $0(0.0)$ \\
\hline $\begin{array}{l}\text { Female to } \\
\text { Male }\end{array}$ & $48(98.0)$ & $0(0.0)$ & $0(0.0)$ & $3(100.0)$ & $0(0.0)$ & $44(100.0)$ & $1(100.0)$ \\
\hline \multicolumn{8}{|l|}{ Marital Status } \\
\hline Married & 126(13.5) & $90(19.8)$ & 19(31.7) & $14(4.0)$ & $1(6.3)$ & $0(0.0)$ & $2(50.00)$ \\
\hline Single & $804(86.5)$ & $364(80.2)$ & $41(68.3)$ & $338(96.0)$ & 15(93.8) & $44(100.0)$ & $2(50.00)$ \\
\hline \multicolumn{8}{|l|}{ Occupation } \\
\hline Unemployed & $568(61.1)$ & 401(88.3) & 15(25.0) & 116(33.0) & $3(18.8)$ & $33(75.0)$ & $0(0.0)$ \\
\hline Employed & $362(38.9)$ & $53(11.7)$ & $45(75.0)$ & $236(67.0)$ & 13(91.3) & $11(25.0)$ & $4(100.0)$ \\
\hline \multicolumn{8}{|l|}{ Income } \\
\hline $\begin{array}{l}\text { Less than } 1 \\
\mathrm{M}^{*}\end{array}$ & $14(4.0)$ & $4(8.0)$ & $2(0.04)$ & $7(0.03)$ & $0(0.0)$ & $1(0.09)$ & $0(0.0)$ \\
\hline $1-2 \mathrm{M}$ & $65(18.0)$ & 16(30.0) & $2(0.04)$ & $43(0.18)$ & $1(0.08)$ & $3(0.28)$ & $0(0.0)$ \\
\hline
\end{tabular}




\begin{tabular}{|c|c|c|c|c|c|c|c|}
\hline & & Key groups & & & & & \\
\hline $2-4 M$ & $147(41.0)$ & $30(57.0)$ & $18(0.4)$ & $88(0.37)$ & $8(0.62)$ & $3(0.28)$ & $0(0.0)$ \\
\hline $\begin{array}{l}\text { More than } 4 \\
\text { M }\end{array}$ & 136(38.0) & $3(6.0)$ & $23(0.51)$ & $98(0.42)$ & $4(0.31)$ & $4(0.37)$ & $4(100.0)$ \\
\hline \multicolumn{8}{|l|}{ Education } \\
\hline $\begin{array}{l}\text { Under } \\
\text { Diploma }\end{array}$ & $255(0.27)$ & $202(0.44)$ & $22(0.37)$ & $21(6.0)$ & $1(6.0)$ & $9(20.0)$ & $0(0.0)$ \\
\hline $\begin{array}{l}\text { Diploma \& } \\
\text { above }\end{array}$ & $675(0.73)$ & $252(0.56)$ & $38(0.63)$ & $331(94.0)$ & 15(94.0) & $35(80.0)$ & $4(100.0)$ \\
\hline \multicolumn{8}{|l|}{ Live with } \\
\hline Homeless & 18(1.9) & $16(3.5)$ & $0(0.0)$ & $2(6.0)$ & $0(0.0)$ & $0(0.0)$ & $0(0.0)$ \\
\hline $\begin{array}{l}\text { Living with a } \\
\text { partner }\end{array}$ & $57(6.1)$ & $26(5.7)$ & $4(6.7)$ & $18(5.1)$ & $2(12.5)$ & $7(15.9)$ & $0(0.0)$ \\
\hline $\begin{array}{l}\text { Living with a } \\
\text { second- } \\
\text { degree } \\
\text { relative }\end{array}$ & $16(1.7)$ & $3(0.7)$ & $5(8.3)$ & $8(2.3)$ & $0(0.0)$ & $0(0.0)$ & $0(0.0)$ \\
\hline $\begin{array}{l}\text { Living with a } \\
\text { first-degree } \\
\text { relative }\end{array}$ & $629(67.6)$ & $315(69.4)$ & $49(81.7)$ & $236(67.0)$ & $10(62.5)$ & 16(36.4) & $3(75.0)$ \\
\hline $\begin{array}{l}\text { Living with } \\
\text { friends }\end{array}$ & $33(3.5)$ & $4(0.9)$ & $0(0.0)$ & $19(5.4)$ & $1(6.3)$ & $8(18.2)$ & $1(25.0)$ \\
\hline Living Alone & 174(18.7) & $88(19.4)$ & $2(3.3)$ & 68(19.3) & $3(18.8)$ & 13(29.5) & $0(0.0)$ \\
\hline Other & $3(0.3)$ & $2(0.4)$ & $0(0.0)$ & $1(0.3)$ & $0(0.0)$ & $0(0.0)$ & $0(0.0)$ \\
\hline
\end{tabular}

Thirty-two percent of respondents had ever heard of HIV testing, of whom 61 percent and slightly more than 20 percent had received information from friends and behavioral diseases counseling centers, respectively. 4 percent of participants had an HIV self-test in the past. When asked about what kind of support they would need to perform the HIV Self-Test, approximately effectively, 50 percent and 23.4 percent of participants indicated a need for the test educational counseling and confirmatory tests, respectively (Table 2 ). 
Table 2

Level of Knowledge about HIV self-test in respondents at baseline by key groups.

\begin{tabular}{|c|c|c|c|c|c|c|c|}
\hline \multirow[t]{3}{*}{ Variables } & Total & FSW & FSW & MSM & MSM & TGs & \multirow{3}{*}{$\begin{array}{l}\text { TGs } \\
\text { Partners } \\
\begin{array}{l}\text { Number } \\
(\%)\end{array}\end{array}$} \\
\hline & & & \multicolumn{2}{|l|}{ Partners } & \multicolumn{2}{|l|}{ Partners } & \\
\hline & $\begin{array}{l}\text { Number } \\
(\%)\end{array}$ & $\begin{array}{l}\text { Number } \\
(\%)\end{array}$ & $\begin{array}{l}\text { Number } \\
(\%)\end{array}$ & $\begin{array}{l}\text { Number } \\
(\%)\end{array}$ & $\begin{array}{l}\text { Number } \\
(\%)\end{array}$ & $\begin{array}{l}\text { Number } \\
(\%)\end{array}$ & \\
\hline \multicolumn{8}{|l|}{ Knowledge about Self-Test } \\
\hline Yes & 298(32.0) & 134(29.5) & $16(26.7)$ & $124(35.2)$ & $9(56.3)$ & $12(27.3)$ & $3(75.0)$ \\
\hline No & $632(68.0)$ & $320(70.5)$ & $44(73.3)$ & $228(64.8)$ & $7(43.8)$ & $32(72.7)$ & $1(25.0)$ \\
\hline \multicolumn{8}{|l|}{$\begin{array}{l}\text { How did you get your HIV test } \\
\text { information? }\end{array}$} \\
\hline Internet & $11(0.04)$ & $0(0.0)$ & $0(0.0)$ & $10(0.08)$ & $0(0.0)$ & $1(0.8)$ & $0(0.0)$ \\
\hline Family & $11(0.04)$ & $4(0.03)$ & $6(0.38)$ & $1(0.01)$ & $0(0.0)$ & $0(0.0)$ & $0(0.0)$ \\
\hline Friends & $186(0.61)$ & $128(0.91)$ & $7(0.44)$ & $44(0.35)$ & $1(0.13)$ & $6(0.50)$ & $0(0.0)$ \\
\hline Social Networks & $18(0.06)$ & $1(0.01)$ & $0(0.0)$ & $15(0.12)$ & $1(0.13)$ & $1(0.08)$ & $0(0.0)$ \\
\hline $\begin{array}{l}\text { Behavioral Diseases Counseling } \\
\text { Center }\end{array}$ & $79(0.26)$ & $7(0.05)$ & $3(0.18)$ & $56(0.44)$ & $6(0.75)$ & $4(0.33)$ & $3(100.0)$ \\
\hline \multicolumn{8}{|l|}{$\begin{array}{l}\text { Have you ever had an HIV self- } \\
\text { test? }\end{array}$} \\
\hline Yes & $38(4.1)$ & $17(3.7)$ & $2(3.3)$ & $16(4.5)$ & $2(12.5)$ & $1(2.3)$ & $0(0.0)$ \\
\hline No & $889(95.6)$ & $437(96.3)$ & $58(96.7)$ & $334(94.9)$ & $14(87.5)$ & $42(95.5)$ & $4(100.0)$ \\
\hline \multicolumn{8}{|l|}{$\begin{array}{l}\text { Which services would you like to } \\
\text { receive after your HIV test? }\end{array}$} \\
\hline Perform confirmatory test & 218(23.4) & $134(129.5)$ & $21(35.0)$ & $58(16.5)$ & $0(0.0)$ & $5(11.4)$ & $0(0.0)$ \\
\hline Educational advice & $471(50.6)$ & $254(55.9)$ & $31(51.7)$ & $163(46.3)$ & $5(31.3)$ & $14(31.8)$ & $4(100.0)$ \\
\hline Psychiatric counseling & $139(14.9)$ & $33(73)$ & $4(6.7)$ & $78(22.2)$ & $5(31.3)$ & 19(43.2) & $0(0.0)$ \\
\hline Other & $102(11.0)$ & $33(7.3)$ & $4(6.7)$ & $53(15.1)$ & $6(37.5)$ & $6(13.6)$ & $0(0.0)$ \\
\hline
\end{tabular}

About 80 percent of participants did their test between 6 am and 6 pm. Virtually 79 percent of participants reported their HIV self-test results in the first 24 hours. Almost 68 percent of the participants reported their test results by visiting counselors at the HIV/AIDS Self-Test centers. A total of 65 percent of the participants stated that they used educational aids to perform the test, of whom 37 percent preferred to use face-to-face counseling when receiving an HIV self-test and 16 percent preferred to use a pamphlet. The average score of satisfaction with teaching aids was very high ( $6.66 \pm 0.65 \mathrm{SD})$. Although only 2 percent of participants used online consulting with experts, the average satisfaction score with online consultants was very high ( $6.86 \pm$ $0.36 \mathrm{SD}$ ) (Table 3). 
Table 3

The use of educational resources and the report of HIV self-test results by key groups.

\begin{tabular}{|c|c|c|c|c|c|c|c|}
\hline Variables & Total & FSW & $\begin{array}{l}\text { FSW } \\
\text { Sexual } \\
\text { Partner }\end{array}$ & MSM & $\begin{array}{l}\text { MSM } \\
\text { Sexual } \\
\text { Partner }\end{array}$ & TG & $\begin{array}{l}\text { TG } \\
\text { Sexual } \\
\text { Partner }\end{array}$ \\
\hline & $\begin{array}{l}\text { Number } \\
(\%)\end{array}$ & $\begin{array}{l}\text { Number } \\
(\%)\end{array}$ & $\begin{array}{l}\text { Number } \\
(\%)\end{array}$ & $\begin{array}{l}\text { Number } \\
(\%)\end{array}$ & $\begin{array}{l}\text { Number } \\
(\%)\end{array}$ & $\begin{array}{l}\text { Number } \\
(\%)\end{array}$ & $\begin{array}{l}\text { Number } \\
(\%)\end{array}$ \\
\hline \multicolumn{8}{|l|}{ When do you take the test? } \\
\hline 6 am to 12 noon & $380(40.9)$ & $205(45.2)$ & $35(58.3)$ & $114(32.4)$ & $9(56.3)$ & $16(36.4)$ & $1(0.25)$ \\
\hline 12 noon to $6 \mathrm{pm}$ & $351(37.7)$ & $217(47.8)$ & $9(15.0)$ & 108(30.7) & $3(18.8)$ & $12(27.3)$ & $2(0.50)$ \\
\hline $6 \mathrm{pm}$ to $12 \mathrm{pm}$ & $165(17.7)$ & $28(6.2)$ & $15(25.0)$ & $106(30.1)$ & $3(18.8)$ & $13(29.5)$ & $0(0.0)$ \\
\hline $12 \mathrm{pm}$ to $6 \mathrm{am}$ & $7(0.08)$ & $2(0.4)$ & $1(1.7)$ & $4(1.1)$ & $0(0.0)$ & $0(0.0)$ & $0(0.0)$ \\
\hline \multicolumn{8}{|l|}{$\begin{array}{l}\text { when did you report the test } \\
\text { result? }\end{array}$} \\
\hline$=<24$ Hours & $689(78.9)$ & $289(63.9)$ & $51(85.0)$ & $294(96.4)$ & $15(100.0)$ & $37(97.4)$ & $3(100.0)$ \\
\hline 24-48 Hours & $114(13.1)$ & 104(23.0) & $6(10.0)$ & $4(1.3)$ & $0(0.0)$ & $0(0.0)$ & $0(0.0)$ \\
\hline$>48$ Hours & $70(8.0)$ & $59(13.1)$ & $3(5.0)$ & $7(2.3)$ & $0(0.0)$ & $1(2.6)$ & $0(0.0)$ \\
\hline \multicolumn{8}{|l|}{$\begin{array}{l}\text { How did you report the test } \\
\text { result? }\end{array}$} \\
\hline the questionnaire link & $1(0.00)$ & $0(0.0)$ & $0(0.0)$ & $0(0.0)$ & $1(6.0)$ & $0(0.0)$ & $0(0.0)$ \\
\hline the online consultant & $9(0.00)$ & $1(0.0)$ & $0(0.0)$ & $7(2.0)$ & $1(6.0)$ & $0(0.0)$ & $0(0.0)$ \\
\hline $\begin{array}{l}\text { Call the counselor at the } \\
\text { HIV/AIDS Self-Test Center }\end{array}$ & $252(0.28)$ & $140(30.9)$ & $24(40.0)$ & $77(23.0)$ & $1(6.0)$ & $10(24.4)$ & $0(0.0)$ \\
\hline $\begin{array}{l}\text { Visit the counselor at the } \\
\text { HIV/AIDS Self-Test Center }\end{array}$ & $610(0.68)$ & $311(68.8)$ & $36(60.0)$ & $220(66.0)$ & 12(80.0) & $28(68.3)$ & $3(100.0)$ \\
\hline $\begin{array}{l}\text { Follow with study the } \\
\text { counselors (inactive follow- } \\
\text { up) }\end{array}$ & $32(0.04)$ & $0(0.0)$ & $0(0.0)$ & $29(8.7)$ & $0(0.0)$ & $3(7.0)$ & $0(0.0)$ \\
\hline \multicolumn{8}{|l|}{$\begin{array}{l}\text { Did you refer to educational } \\
\text { aids (site, pamphlet, etc.) to } \\
\text { complete your information? }\end{array}$} \\
\hline Yes & $607(67.0)$ & $343(75.9)$ & $36(60.0)$ & $196(57.8)$ & $9(60.0)$ & $22(54.0)$ & 1(33.3) \\
\hline No & $303(33.0)$ & $109(24.11)$ & $24(40.0)$ & $143(42.2)$ & $6(40.0)$ & $19(46.3)$ & $2(66.7)$ \\
\hline \multicolumn{8}{|l|}{$\begin{array}{l}\text { Which teaching aid do you } \\
\text { prefer to use? }\end{array}$} \\
\hline Pamphlet & $149(25.0)$ & $59(17.2)$ & 7(19.4) & $72(37.0)$ & $1(11.0)$ & $10(46.0)$ & $0(0.0)$ \\
\hline $\begin{array}{l}\text { In-person counseling when } \\
\text { receiving a test }\end{array}$ & $346(57.0)$ & $241(70.2)$ & $18(50.0)$ & $73(37.0)$ & $6(67.0)$ & $7(32.0)$ & $1(100.0)$ \\
\hline $\begin{array}{l}\text { Website and educational } \\
\text { video }\end{array}$ & $98(16.0)$ & $41(11.9)$ & $10(28.0)$ & $41(21.0)$ & $2(22.0)$ & $4(18.0)$ & $0(0.0)$ \\
\hline Other & $14(2.0)$ & $2(0.0)$ & $1(3.0)$ & $10(5.0)$ & $0(0.0)$ & $1(5.0)$ & $0(0.0)$ \\
\hline $\begin{array}{l}\text { Did you call the introduced } \\
\text { expert online for more } \\
\text { information or a question? }\end{array}$ & & & & & & & \\
\hline
\end{tabular}




\begin{tabular}{|llllllll|}
\hline Variables & Total & FSW & $\begin{array}{l}\text { FSW } \\
\text { Sexual } \\
\text { Partner }\end{array}$ & MSM & $\begin{array}{l}\text { MSM } \\
\text { Sexual } \\
\text { Partner }\end{array}$ & TG & $\begin{array}{l}\text { TG } \\
\text { Sexual } \\
\text { Partner }\end{array}$ \\
\hline Yes & $14(2.0)$ & $0(0.0)$ & $1(1.7)$ & $11(3.0)$ & $1(6.6)$ & $1(2.4)$ & $0(0.0)$ \\
\hline No & $896(98.0)$ & $452(100.0)$ & $59(98.3)$ & $328(96.8)$ & $14(93.3)$ & $40(97.6)$ & $3(100.0)$ \\
\hline $\begin{array}{l}\text { Were you satisfied with the } \\
\text { introduced expert online? }\end{array}$ & $6.86(0.36)$ & - & $6.0(0.0)$ & $7.0(0.0)$ & $7.0(0.0)$ & $6.0(0.0)$ & - \\
\hline $\begin{array}{l}\text { Were you satisfied with the } \\
\text { teaching aids? }\end{array}$ & $6.66(0.65)$ & $6.73(0.57)$ & $6.75(0.50)$ & $6.57(0.72)$ & $6.22(1.39)$ & $6.41(0.73)$ & $6.00(0.0)$ \\
\hline
\end{tabular}

The approval of the use of HIV self-tests in Iranian high-risk groups was significantly high so that 99 percent of participants in response to the question that "in general, was it acceptable for you to perform the test in this way?" answered yes and 98 percent of them said they would like to retake this test. Ninety-one percent of the participants also stated that they would recommend this test to their friends and sexual partners. Sixty-seven percent of participants preferred to take the test with a reliable consultant, and 60 percent preferred taking the test alone. In response to the question "where do you want to get the test?", $51 \%, 48 \%, 45 \%$, and $37 \%$ of the participants chose drug stores, behavioral diseases counseling centers, comprehensive health service centers, and online stores, respectively (Table 4). 
Results of Acceptability of the use of HIV Self-Test in Iranian key groups

\begin{tabular}{|c|c|c|c|c|c|c|c|}
\hline Variables & Total & FSW & $\begin{array}{l}\text { FSW } \\
\text { Sexual } \\
\text { Partner }\end{array}$ & MSM & $\begin{array}{l}\text { MSM } \\
\text { Sexual } \\
\text { Partner }\end{array}$ & TG & $\begin{array}{l}\text { TG } \\
\text { Sexual } \\
\text { Partner }\end{array}$ \\
\hline & $\begin{array}{l}\text { Number } \\
(\%)\end{array}$ & $\begin{array}{l}\text { Number } \\
(\%)\end{array}$ & $\begin{array}{l}\text { Number } \\
(\%)\end{array}$ & $\begin{array}{l}\text { Number } \\
(\%)\end{array}$ & $\begin{array}{l}\text { Number } \\
(\%)\end{array}$ & $\begin{array}{l}\text { Number } \\
(\%)\end{array}$ & $\begin{array}{l}\text { Number } \\
(\%)\end{array}$ \\
\hline \multicolumn{8}{|l|}{$\begin{array}{l}\text { In general, was it acceptable for } \\
\text { you to perform the test in this } \\
\text { way? }\end{array}$} \\
\hline Yes & $903(99.2)$ & 450(99.9) & $60(100.0)$ & $335(98.8)$ & $15(100.0)$ & $40(98.0)$ & $3(100.0)$ \\
\hline No & $7(0.8)$ & $2(1.0)$ & $0(0.0)$ & $4(1.00)$ & $0(0.0)$ & $1(2.00)$ & $0(0.0)$ \\
\hline \multicolumn{8}{|l|}{$\begin{array}{l}\text { Would you like to use this test } \\
\text { again? }\end{array}$} \\
\hline Yes & 881(98.4) & 447(99.1) & $6(100.0)$ & $324(98.8)$ & 14(93.3) & $33(86.8)$ & $3(100.0)$ \\
\hline No & $14(1.5)$ & $4(0.9)$ & $0(0.0)$ & $4(1.2)$ & $1(6.7)$ & $5(13.1)$ & $0(0.0)$ \\
\hline \multicolumn{8}{|l|}{$\begin{array}{l}\text { In whose presence do you prefer } \\
\text { to take the test? * }\end{array}$} \\
\hline Alone & $550(60.0)$ & $297(66.0)$ & $34(57.0)$ & $189(56.0)$ & $10(67.0)$ & $18(44.0)$ & $2(50.0)$ \\
\hline A Family member & $75(8.0)$ & $65(14.0)$ & $1(2.0)$ & $7(2.0)$ & $0(0.0)$ & $2(5.0)$ & $0(0.0)$ \\
\hline A Friend & $162(18.0)$ & $72(16.0)$ & $5(2.0)$ & $66(19.0)$ & $4(27.0)$ & $15(37.0)$ & $0(0.0)$ \\
\hline A reliable consultant & $607(67.0)$ & $317(70.0)$ & $37(2.6)$ & $218(64.0)$ & 11(73.0) & $22(54.0)$ & $2(50.0)$ \\
\hline Others & $4(0.0)$ & $0(00.0)$ & $0(2.0)$ & $3(1.0)$ & $0(0.0)$ & $1(2.0)$ & $0(0.0)$ \\
\hline \multicolumn{8}{|l|}{$\begin{array}{l}\text { Where do you want to get self- } \\
\text { test? * }\end{array}$} \\
\hline Drug store & $464(51.0)$ & $241(53.0)$ & $31(52.0)$ & $169(50.0)$ & $6(40.0)$ & $15(37.0)$ & $2(67.0)$ \\
\hline Online store & $337(37.0)$ & 177(39.0) & $14(23.0)$ & 127(37.0) & $6(40.0)$ & $13(32.0)$ & $0(0.0)$ \\
\hline $\begin{array}{l}\text { Behavioral Diseases Counseling } \\
\text { Center }\end{array}$ & $441(48.0)$ & 138(31.0) & 18(30.0) & $247(73.0)$ & 12(80.0) & $23(56.0)$ & $3(100.0)$ \\
\hline $\begin{array}{l}\text { Comprehensive health service } \\
\text { centers }\end{array}$ & $409(45.0)$ & $321(71.0)$ & $22(37.0)$ & $60(18.0)$ & $2(13.0)$ & $4(10.0)$ & $0(0.0)$ \\
\hline Stamping Ground & 115(13.0) & $41(9.0)$ & $7(12.0)$ & $58(17.0)$ & $0(0.0)$ & $8(20.0)$ & 1(33.0) \\
\hline Peer & $124(14.0)$ & $8(2.0)$ & $2(3.0)$ & $100(29.0)$ & $2(13.0)$ & $12(29.0)$ & $0(0.0)$ \\
\hline Others & 19(2.0) & $14(3.0)$ & $1(2.0)$ & $4(1.0)$ & $0(0.0)$ & $0(00.0)$ & $0(0.0)$ \\
\hline \multicolumn{8}{|l|}{$\begin{array}{l}\text { Do you recommend this test to } \\
\text { your other friends or partners? }\end{array}$} \\
\hline Yes & 828(91.0) & $399(88.0)$ & $53(88.0)$ & $323(95.0)$ & 14(93.0) & $36(88.0)$ & $3(100.0)$ \\
\hline No & $25(3.0)$ & $10(2.0)$ & $1(2.0)$ & $11(4.0)$ & $0(0.0)$ & $3(7.0)$ & $0(0.0)$ \\
\hline I don't know & $57(6.0)$ & $43(10.0)$ & $6(10.0)$ & $5(1.0)$ & $1(7.0)$ & $2(5.0)$ & $0(0.0)$ \\
\hline
\end{tabular}




\section{Reactive Participants And Connecting Them To The Counseling Centers}

Overall, 14 participants (1.5\%) had reactive outcomes, of whom 10, 3, and 1 person were in the key groups of MSM, TGs, and FSWs, respectively. They referred to the behavioral diseases counseling centers for confirmatory tests. The confirmatory test of the two participants was negative. Individuals were introduced to the health center with a confirmed test to receive services (Table 5).

Table 5

Reactive participants and Connecting to the counseling center

\begin{tabular}{|lc|}
\hline Variables & Number (\%) \\
\hline What was the HIV self-test result? & 888(95.4) \\
\hline Negative & $14(1.5)$ \\
\hline Positive (Reactive) & $28(3.1)$ \\
\hline No answer/ No Response & \\
\hline In positive participants, would you like to go to a trusted counseling center for a confirmatory test? & $12(86)$ \\
\hline Yes & $2(14)$ \\
\hline No/ No Answer & \\
\hline In positive participants, would you like to talk to a trusted expert before having a confirmation test? & $10(71)$ \\
\hline Yes & $4(29)$ \\
\hline No Answer/ No Response & \\
\hline
\end{tabular}

\section{Feasibility of the use of HIV Self-Tests by key Groups}

The feasibility of the use of HIV self-tests in Iranian high-risk groups was significantly high. Ninety-seven percent of participants did not have any confidentiality problems while preparing or performing the test. In general, feasibility was assessed based on five questions. The overall feasibility score was 6.33 (0.824 SD). Totally, in response to the question of "how accurate is the test result?", participants gave an average of 6.10 out of 7 points. Taking tests, reading HIV test results, finding a safe place to do the test, and accessing HIV self-testing showed a high average (Table 6). 
Table 6

Results of Feasibility of the use of HIV Self-Test by key Groups

\begin{tabular}{|c|c|c|c|c|c|c|c|}
\hline & FSW & $\begin{array}{l}\text { FSW } \\
\text { Sexual } \\
\text { Partner }\end{array}$ & MSM & $\begin{array}{l}\text { MSM } \\
\text { Sexual } \\
\text { Partner }\end{array}$ & TG & $\begin{array}{l}\text { TG } \\
\text { Sexual } \\
\text { Partner }\end{array}$ & Total \\
\hline Questions & $\begin{array}{l}\text { Mean } \\
\text { (SD) }\end{array}$ & $\begin{array}{l}\text { Mean } \\
\text { (SD) }\end{array}$ & $\begin{array}{l}\text { Mean } \\
\text { (SD) }\end{array}$ & Mean (SD) & $\begin{array}{l}\text { Mean } \\
\text { (SD) }\end{array}$ & $\begin{array}{l}\text { Mean } \\
(\mathrm{SD})\end{array}$ & $\begin{array}{l}\text { Mean } \\
(\mathrm{SD})\end{array}$ \\
\hline How accurate is the test result? & $\begin{array}{l}6 / 17 \\
(1 / 058)\end{array}$ & $\begin{array}{l}6 / 13 \\
(1 / 016)\end{array}$ & $\begin{array}{l}6 / 00 \\
(1 / 179)\end{array}$ & $\begin{array}{l}5 / 73 \\
(1 / 387)\end{array}$ & $\begin{array}{l}6 / 10 \\
(0 / 800)\end{array}$ & $\begin{array}{l}6 / 33 \\
(0 / 577)\end{array}$ & $\begin{array}{l}6.10 \\
(1.098)\end{array}$ \\
\hline $\begin{array}{l}\text { How easy was it for you to access your } \\
\text { HIV test? }\end{array}$ & $\begin{array}{l}5 / 70 \\
(1 / 872)\end{array}$ & $\begin{array}{l}5 / 28 \\
(2.293)\end{array}$ & $\begin{array}{l}6 / 28 \\
(1 / 141)\end{array}$ & $\begin{array}{l}5 / 67 \\
(1.496)\end{array}$ & $\begin{array}{l}6 / 32 \\
(1 / 192)\end{array}$ & $\begin{array}{l}5 / 67 \\
(1 / 528)\end{array}$ & $\begin{array}{l}5.92 \\
(1.665)\end{array}$ \\
\hline $\begin{array}{l}\text { How easy was it for you to get tested } \\
\text { for HIV yourself? }\end{array}$ & $\begin{array}{l}6 / 42 \\
(1 / 134)\end{array}$ & $\begin{array}{l}6 / 37 \\
(1.089)\end{array}$ & $\begin{array}{l}6 / 74 \\
(0 / 573)\end{array}$ & $\begin{array}{l}6 / 73 \\
(0.594)\end{array}$ & $\begin{array}{l}6 / 78 \\
(0 / 571)\end{array}$ & $\begin{array}{l}7 / 00 \\
(0 / 000)\end{array}$ & $\begin{array}{l}6.56 \\
(0.941)\end{array}$ \\
\hline $\begin{array}{l}\text { How easy was it for you to read the } \\
\text { test results of the HIV test itself? }\end{array}$ & $\begin{array}{l}6 / 44 \\
(1 / 136)\end{array}$ & $\begin{array}{l}6 / 35 \\
(1.132)\end{array}$ & $\begin{array}{l}6 / 74 \\
(0 / 605)\end{array}$ & $\begin{array}{l}6 / 87 \\
(0.352)\end{array}$ & $\begin{array}{l}6 / 61 \\
(0 / 666)\end{array}$ & $\begin{array}{l}7 / 00 \\
(0 / 000)\end{array}$ & $\begin{array}{l}6.56 \\
(0.951)\end{array}$ \\
\hline $\begin{array}{l}\text { How easy was it for you to find a safe } \\
\text { place to test? }\end{array}$ & $\begin{array}{l}6 / 59 \\
(0 / 855)\end{array}$ & $\begin{array}{l}6 / 10 \\
(1.285)\end{array}$ & $\begin{array}{l}6.50 \\
(0 / 905)\end{array}$ & $\begin{array}{l}6 / 47 \\
(0.743)\end{array}$ & $\begin{array}{l}6 / 56 \\
(0 / 867)\end{array}$ & $\begin{array}{l}6 / 67 \\
(0 / 577)\end{array}$ & $\begin{array}{l}6.52 \\
(0.911)\end{array}$ \\
\hline Total & $\begin{array}{l}6.26 \\
(0.960)\end{array}$ & $\begin{array}{l}6.05 \\
(1.001)\end{array}$ & $\begin{array}{l}6.45 \\
(0.569)\end{array}$ & $\begin{array}{l}6.29 \\
(0.692)\end{array}$ & $\begin{array}{l}6.47 \\
(0.588)\end{array}$ & $\begin{array}{l}6.53 \\
(0.503)\end{array}$ & $\begin{array}{l}6.33 \\
(0.824)\end{array}$ \\
\hline
\end{tabular}

\section{Qualitative Results}

6 to 8 key groups (MSM, FSWs, and TGs) were selected separately, and interviews were conducted with each population as a focused group discussion (FGD). All results were analyzed using content analysis and shown in Table 7. After interviewing and analyzing the findings, the main challenges in distributing and accessing the HIVST, consulting on how to perform the HIVST, announcing the HIVST result, and connecting to health care centers were summarized (Table 7). Proposed solutions to address these challenges by key groups (MSM, FSWs, and TGs) were presented in Table 7. 
The result of qualitative study analysis (Challenges of HIV self-testing and proposed solutions)

\begin{tabular}{|c|c|}
\hline Challenges & Proposed Solutions \\
\hline \multirow{9}{*}{$\begin{array}{l}\text { Distribution and } \\
\text { access to HIVST }\end{array}$} & Presentation of the HIVST in selected centers (currently, this method is suitable): \\
\hline & $\begin{array}{l}\text { - Selecting centers with appropriate space and maintaining confidentiality, with the help of trained } \\
\text { experts and peers }\end{array}$ \\
\hline & - Access to these centers should be as easy as possible. \\
\hline & Presentation of the HIVST in hangouts: \\
\hline & -Distribution of the HIVST by peers in hangouts and key population gathering places \\
\hline & -Establishing the presentation HIVST centers in places close to hangouts \\
\hline & Presentation of the HIVST in pharmacy \\
\hline & Presentation of the HIVST in the online store \\
\hline & Eliminate legal barriers to distribution or access to HIVST \\
\hline \multirow{6}{*}{$\begin{array}{l}\text { Counseling on } \\
\text { how to perform } \\
\text { the HIVST }\end{array}$} & In-person or Face to Face consultation: \\
\hline & $\begin{array}{l}\text {-Setting up or developing centers for the distribution of HIVST with the presence of trained experts or } \\
\text { using trained peers to provide sufficient and complete explanations about HIVST, how to do and } \\
\text { receive results }\end{array}$ \\
\hline & -More emphasis on how to perform the HIVST and how to read the results \\
\hline & Online counseling \\
\hline & -Launching training webinars and online training websites with experts or trained peers \\
\hline & $\begin{array}{l}\text {-Preparation and distribution of pamphlets and educational brochures during HIVST distribution or } \\
\text { online for key groups }\end{array}$ \\
\hline \multirow{2}{*}{$\begin{array}{l}\text { How to do the } \\
\text { HIVST }\end{array}$} & - Provide appropriate and sufficient training on how to perform the HIVST \\
\hline & - More appropriate distribution of educational materials related to how to perform HIVST \\
\hline \multirow{3}{*}{$\begin{array}{l}\text { Announcing the } \\
\text { HIVST result }\end{array}$} & -Provide test results online (offline) or in person if a suitable platform is prepared \\
\hline & - Setting up the monitoring system for key groups after receiving HIVST \\
\hline & $\begin{array}{l}\text { - Setting up a system of psychological and psychiatric counseling for key groups (online or in-person) } \\
\text { after receiving the HIVST results }\end{array}$ \\
\hline \multirow{4}{*}{$\begin{array}{l}\text { Connect to } \\
\text { treatment care } \\
\text { centers }\end{array}$} & -Providing appropriate training to gain the trust of key groups to refer health care centers \\
\hline & -Providing incentives and gifts for referring to health care centers \\
\hline & - Setting up health care centers in the closest and most accessible places for key groups \\
\hline & -Providing free or low-cost medical services in health care centers \\
\hline
\end{tabular}

\section{Discussion}

This study aimed to evaluate the feasibility of using rapid HIV self-tests in high-risk populations. The results showed high Acceptance of HIV self-tests (HIVST) in key groups such as MSM, FSWs, and TGs in Iran. On the other hand, most of the key groups participating in the present study, such as TGs, MSM, and FSWs, stated that it was easy to perform the test. They accepted the way the test was presented, the Facility-Based Method. The present study results were entirely in line with the results of previous studies [20-25]. 
More than 20 countries worldwide have accepted the policies concerning HIVST, announced by the World Health Organization. They have taken the necessary measures to implement this test in their own country. HIVST is currently presented in different ways in the world. The first method is to go to clinics to do the test (Clinic-Based Testing or CBT) or to provide the test from private or public pharmacies, which is called the Facility-Based Method. In this method, whole groups, by referring to selected centers and receiving face-to-face consultations along with pamphlets and training brochures to perform the test, receive the test and take action to do it in the center or at their home. The second way of presenting HIVST worldly is access to the tests in public places, such as buses, subway, train stations, public hospitals, or shops across the city, province, or country. This method is known as the Community Based Method. In this way, key groups can receive and perform the test anytime and anywhere. The third method to present HIVST to key groups is to visit reputable websites, purchase and order tests online. This method is known as Online Shopping [25-30].

According to the results of this study in Iran, key groups have considered the Facility-Based Method better to receive HIVST. This result was confirmed by the results of previous studies, while most studies have used the Facility-Based Method to distribute and present HIVST [31-33]. The qualitative interview conducted in the present research showed that from the perspective of most key groups, the best and easiest way to access HIVST in the country would be to provide tests in service delivery centers such as behavioral diseases counseling and other related centers. Of course, other possible methods in Iran, such as offering tests in hangouts, pharmacies and providing the necessary facilities for online test sales, can also be helpful.

A notable result in the present study was the knowledge of key groups and their partners on HIVST. The results showed that $68 \%$ of the key groups in the study did not have sufficient knowledge about HIVST. The results were similar for the different vital groups participating in the study, and all key groups and their partners had insufficient information and knowledge about HIVST. Based on these results, it is necessary to design and implement an accurate and easy plan to increase the knowledge of these groups and their partners in the country. These programs can be done with inter-sectorial cooperation and the cooperation of the key groups. Key individuals and their partners can play an instrumental and influential role in increasing other key groups' awareness and knowledge related to HIVST. According to this research, most key groups participating in this study have received little information about HIVST from their friends. Also, to increase the awareness of critical groups, the results of qualitative interviews in this research showed that the whole groups were more focused on in-person and in-absentia counseling. These people emphasized that centers for distributing self-tests should be set up with qualified and trained experts or the key people themselves to provide complete training related to self-tests. These groups also believed that holding workshops and webinars online and in-person connected with HIVST could significantly raise awareness. These methods can be effective in increasing the key groups' awareness and knowledge related to HIVST. The results of previous studies have also emphasized these methods to increase the awareness of critical people [31-37]. For example, Tucker JD et al. have noted that social marketing can be very effective in training people about receiving and performing HIVST and evaluating their results [32]. In addition, by promoting the advantages of this test over effectively train better preservation of the confidentiality and secrets of key groups, and the absence of blood or blood sampling for this test's advantages over effectively training HIVST [34-37].

At present, in none of the countries, a specific policy and plan have not been developed and designed for the process after the test, and the taken actions are scattered and irregular. A limited number of countries, such as the United States or the European countries, have hired several trained experts. These experts follow up test results in key groups using fixed or mobile phone numbers (the passive method) or in-person sessions (the active method) and ask these people to go to service centers to continue the process and receive medical services. This method was also used in the present study, and the entire groups were satisfied with this process. Therefore, it can be concluded that the Facility-Based Method and actively and passively following the results is the most appropriate method for presenting HIVST in Iran.

Based on the results of this study, it can be concluded that HIVST can be presented to the general population, especially the key groups of MSM, FSWs, and TGs, or other key groups such as PWID, prisoners, etc. With this test, we can even reach the key groups who have not been before tested for HIV at all because most of the key participants in the present study mentioned that they had never been tested for HIV before. In Iran, regular and specific services related to HIV are not provided to key groups currently, especially to transgender people and MSM or FSWs. So, the distribution of HIVST in these groups can be a valuable and practical step towards preventing or controlling HIV infection in them.

Page $14 / 18$ 
This research was the first study in Iran to evaluate the possibility of HIVST in critical groups of MSM, TGs, and FSWs. The study also focused on the groups most at risk for HIV. One of the limitations of this study has been the low sample size of the Transgender group, so it is necessary to conduct further studies with larger sample sizes in this group about using HIVST. In this study, through the Welfare Organization, non-governmental organizations, the Ministry of Health and Medical Education, and finally through the peers of the transgender groups, invited to receive HIVST. However, a small number of these people referred to receive them. A variety of reasons may be considered for this, such as the corona pandemic, the transgender groups' fear and anxiety regarding the infection detection by HIVST, the stigma or discrimination in selected centers when receiving HIVST, and lack of transgender groups' trust in HIVST providers in selected centers. Nevertheless, the results related to the TG group showed that they were satisfied with HIVST. On the other hand, this study was a pilot one to investigate the feasibility of using HIVST in the whole groups; therefore, further studies are needed to be separately done in these groups, and other studies with larger sample sizes can be designed and implemented in other key groups such as PWID, prisoners or even the general population.

\section{Conclusion}

HIVST in the groups of FSWs and MSM had suitable Acceptability according to the present study results, and the Acceptance of this test was good in these groups. However, more appropriate and accurate planning for better access of these groups to HIVST is needed in the community on how to provide and distribute the test, how to provide appropriate training related to the test, how to follow people's test results, and finally, how to refer and link these people to care and treatment centers. In Iran, these groups are less likely than other populations to go to service centers to do HIV self-tests and receive services because there is still stigma and discrimination related to HIV in Iran. The distribution and feasibility study of HIVST in key groups or atrisk populations can help reach out to people who do not refer for testing because of stigma, discrimination, or fear of disclosure.

\section{Abbreviations}

HIVST

HIV self-testing

FSW

female sex workers

TG

transgender

MSM

men who have sex with men

UN

united nations

SD

standard deviation

IDUs

Injecting Drug Users.

\section{Declarations}

\section{- Ethics approval and consent to participate}

This study has been received the national ethical code (Ethical code: IR.MUK.REC.1399.133). Also, all participants (MSM, FSWs, and TGs) signed an informed consent form.

\section{- Consent for publication}


Not applicable

\section{- Availability of data and materials}

The datasets generated and analyzed during the current study are not publicly available due to their sensitive and potentially personally identifiable nature. However, they are available from the corresponding author on reasonable request.

\section{- Competing interests}

All authors declare that they have no competing interests.

\section{- Funding}

This research received no external funding.

\section{- Authors' contributions}

Conceptualization: YM, and GM; methodology: YM, and LM; software: YM; validation: PAK, AV, KT, and YM; Qualitative data collection and analysis: EEA, and YM; investigation: AV, KT, PAK; and LM, resources, YM; data curation, YM, and EEA, writingreview and editing: GM, YM, and EEA; supervision: GM; project administration: GM. All authors have read and agreed to the published version of the manuscript.

\section{- Acknowledgments}

Not applicable

\section{References}

1. Cassels, S., Time, population mobility, and HIV transmission. The Lancet HIV, 2020.

2. Chang, Y.M. et al., HIV self-testing in Pune, India: perspectives and recommendations of female sex workers and peer educators. AIDS care, 2020. 32(2): p. 182-185.

3. Etemad, K., et al., Prevalence of HIV infection and associated risk behaviors among people who inject drugs in Kermanshah, Iran: 2017. Journal of Substance Use, 2020: p. 1-4.

4. Khezri, M., et al., Prevalence of substance use and associated risk factors among homeless youth in Iran: a cross-sectional study. Children and Youth Services Review, 2020: p. 105070.

5. Mazaheri-Tehrani, E., et al., Prevalence of HIV-associated Neurocognitive Disorder (HAND) and its subgroups among HIVpositive persons on antiretroviral therapy in Iran. Psihologija, 2020(00): p. 1-1.

6. Pourjam, R., et al., An Analytical Comparison of Knowledge, Attitudes, and Practices Regarding HIV/AIDS Among Medical and Non-Medical Students in Iran. HIV/AIDS (Auckland, NZ), 2020. 12: p. 165.

7. Bingham, C.C.J., A. Barbosa, and B. Grinsztejn, Internet-based HIV self-testing program to increase HIV testing uptake for men who have sex with men in Brazil.

8. De Boni, R.B., et al., An internet-based HIV self-testing program to increase HIV testing uptake among men who have sex with men in Brazil: Descriptive cross-sectional analysis. Journal of medical Internet research, 2019. 21(8): p. e14145.

9. den Daas, C., et al., Increasing awareness and prompting HIV testing: Contributions of Amsterdam HIV Testing Week 2016. International Journal of STD \& AIDS, 2018. 29(11): p. 1057-1065.

10. Devillé, W. and H. Tempelman, Feasibility and robustness of an oral HIV self-test in a rural community in South Africa: An observational diagnostic study. PloS one, 2019. 14(4): p. e0215353.

11. Edelstein, Z.R., et al., Five Waves of an Online HIV Self-Test Giveaway in New York City, 2015 to 2018. Sexually Transmitted Diseases, 2020. 47(5S): p. S41-S47. 
12. Estem, K.S., J. Catania, and J.D. Klausner, HIV self-testing: a review of current implementation and fidelity. Current HIV/AIDS Reports, 2016. 13(2): p. 107-115.

13. Chen, Y.-H., et al., Increases in HIV status disclosure and sexual communication between South African men who have sex with men and their partners following use of HIV self-testing kits. AIDS care, 2020: p. 1-8.

14. Colfax, G., et al., What happened to home HIV test collection kits? Intent to use kits, actual use, and barriers to use among persons at risk for HIV infection. AIDS care, 2002. 14(5): p. 675-682.

15. Daniels, J., et al., Will men who have sex with men use short-messaging services to send photos of completed HIV selftests to researchers? Journal of the Association of Nurses in AIDS Care, 2016. 27(5): p. 722-726.

16. Napierala, S., et al., Male partner testing and sexual behavior following provision of multiple HIV self-tests to Kenyan women at higher risk of HIV infection in a cluster randomized trial. Journal of the International AIDS Society, 2020. 23: p. e25515.

17. Napierala, S., et al., HIV self-testing services for female sex workers, Malawi and Zimbabwe. Bulletin of the World Health Organization, 2019. 97(11): p. 764.

18. Iribarren, S., et al., Using an HIV self-test kit to test a partner: Attitudes and preferences among high-risk populations. AIDS and Behavior, 2020. 24(11): p. 3232-3243.

19. Liang, T.S., et al., Rapid HIV testing of clients of a mobile STD/HIV clinic. AIDS patient care \& STDs, 2005. 19(4): p. 253257.

20. Lippman, S.A., et al., High Acceptability and increased HIV testing frequency following introduction of HIV self-testing and network distribution among South African MSM. Journal of acquired immune deficiency syndromes (1999), 2018. 77(3): p. 279.

21. Lippman, S.A., et al., Acceptability and feasibility of HIV self-testing among transgender women in San Francisco: a mixedmethods pilot study. AIDS and Behavior, 2016. 20(4): p. 928-938.

22. Krause, J., et al., Acceptability of HIV self-testing: a systematic literature review. BMC public health, 2013. 13(1): p. 1-9.

23. Lee, V.J., et al., User acceptability and feasibility of self-testing with HIV rapid tests. JAIDS Journal of Acquired Immune Deficiency Syndromes, 2007. 45(4): p. 449-453.

24. Njau, B., et al., feasibility of an HIV self-testing intervention: a formative qualitative study among individuals, community leaders, and HIV testing experts in northern Tanzania. BMC Public Health, 2020. 20(1): p. 1-15.

25. Ng, OT, et al., Accuracy and user-acceptability of HIV self-testing using an oral fluid-based HIV rapid test. 2012.

26. Pant Pai, N., et al., Supervised and Unsupervised Self-Testing for HIV in High-and Low-Risk Populations. 2013.

27. Pant Pai, N., et al., Supervised and unsupervised self-testing for HIV in high-and low-risk populations: a systematic review. PLoS medicine, 2013. 10(4): p. e1001414.

28. Eshun-Wilson, I., et al., A Systematic Review and Network Meta-analyses to Assess the Effectiveness of Human Immunodeficiency Virus (HIV) Self-testing Distribution Strategies. Clinical Infectious Diseases, 2021.

29. Hensen, B., et al., Who accepts and who uses community-based secondary distribution HIV self-testing (HIVST) kits? Findings from the intervention arm of a cluster-randomized trial of HIVST distribution nested in four HPTN 071 (POPART) communities in Zambia. Journal of acquired immune deficiency syndromes (1999), 2020. 84(4): p. 355.

30. Kumwenda, M.K., et al., Exploring social harms during distribution of HIV self-testing kits using mixed-methods approaches in Malawi. Journal of the International AIDS Society, 2019. 22: p. e25251.

31. Lightfoot, M.A., et al., Using a social network strategy to distribute HIV self-test kits to African American and Latino MSM. JAIDS Journal of Acquired Immune Deficiency Syndromes, 2018. 79(1): p. 38-45.

32. Lyons, C.E., et al., Use and Acceptability of HIV self-testing among first-time testers at risk for HIV in Senegal. AIDS and Behavior, 2019. 23(2): p. 130-141.

33. Tucker, J.D., et al., HIV self-testing among key populations: an implementation science approach to evaluating self-testing. Journal of virus eradication, 2015. 1(1): p. 38-42. 
34. Chipungu, J., et al., Understanding linkage to care with HIV self-test approach in Lusaka, Zambia-A mixed-method approach. PLoS One, 2017. 12(11): p. e0187998.

35. Choko, AT, et al., A pilot trial of the peer-based distribution of HIV self-test kits among fishermen in Bulisa, Uganda. PloS one, 2018. 13(11): p. e0208191.

36. Conserve, DF, et al., Sexual and social network correlates of willingness to self-test for HIV among ever-tested and nevertested men: implications for the Tanzania STEP project. AIDS care, 2019. 31(2): p. 169-176.

37. Matovu, J.K., et al., "If I had not taken it [HIVST kit] home, my husband would not have come to the facility to test for HIV": HIV self-testing perceptions, delivery strategies, and post-test experiences among pregnant women and their male partners in Central Uganda. Global health action, 2018. 11(1): p. 1503784. 